\title{
MENSURAÇÃO DO DESEMPENHO CONTÁBIL-FINANCEIRO: COMPARANDO METODOLOGIAS DE CONSOLIDAÇÃO DE ÍNDICES
}

\author{
Marcelo Alvaro da Silva Macedo \\ FACC/UFRJ \\ Doutorado em Engenharia de Produção pela COPPE-UFRJ \\ tmalvaro.facc.ufrj@gmail.com \\ Luiz João Corrar \\ Doutorado em Controladoria e Contabilidade pela USP \\ ljcorrar@usp.br
}

Recebido em 06/setembro/2010
Aprovado em 18/agosto/2012

\section{RESUMO}

Este artigo tem como objetivo comparar o desempenho organizacional sob a perspectiva contábil-financeira, com base nos índices do ranking Melhores e Maiores (MM) da FIPECAFI/EXAME, da Análise Envoltória de Dados (DEA) e da Análise Hierárquica (AHP), como instrumentos de consolidação de vários vetores de desempenho em um único indicador de natureza multicriterial. Os resultados a priori mostram que, de maneira geral, os rankings apresentam diferenças, pois as empresas não obtêm as mesmas posições nas três metodologias. Somente em relação aos rankings MM e AHP é que se observa uma fraca correlação (aproximadamente 0,50), com significância bem próxima a 10\%. Porém, com base na medida de concordância W de Kendall, observa-se que os três rankings possuem associação significativa. Com apoio nesses resultados, pode-se afirmar que as diferenças metodológicas, entre os rankings obtidos, foram fracamente capturadas pela análise de correlação, porém não foram significativas para a medida de concordância utilizada, que sugere uma convergência entre os rankings formados. Palavras-chaves: Desempenho Organizacional; Índices Contábil Financeiros; Análise Multicrédito; DEA; AHP.

\begin{abstract}
This article aims to compare organizational performance under the accounting and financial perspective, with the indexes of Melhores e Maiores (MM) ranking of FIPECAFI/EXAME, Data Envelopment Analysis (DEA) and Analytic Hierarchy Process (AHP), as instruments for consolidating multiples vectors into a single multi-criteria performance indicator. The results show that a priori, in general, the rankings differ, because companies do not get the same positions in the three methodologies. Only in relation to MM and AHP rankings there is a weak correlation (approximately 0.50), with significance at around 10\%. However, based on the measure of concordance W Kendall, it notes that the three rankings have a significant association. Based on these results, it can be argued that the methodological differences, between the obtained rankings, were poorly captured by correlation analysis, but were not significant for the used measure of concordance, suggesting a convergence between the formed rankings.
\end{abstract}

Key words: Organizational Performance. Accounting and Financial Ratios. Multicriterial Analysis. DEA. AHP 


\section{INTRODUÇão}

A análise do desempenho organizacional é um dos aspectos que vêm governando, em maior ou menor grau, dependendo da organização e do setor, a atuação de empresas no Brasil e no mundo, nos últimos anos. Isso porque é por meio dela que a administração da organização se torna capaz de monitorar, comparar e, até mesmo, corrigir o desempenho corporativo, sendo, assim, fundamental para a sobrevivência da organização num ambiente competitivo.

Apesar disso, a análise do desempenho organizacional é sempre algo passivo de muitas discussões. Questionamentos sobre quais indicadores utilizar e como consolidá-los de forma a estabelecer um critério justo de avaliação de performance estão presentes em vários estudos e no dia a dia das empresas.

De acordo com Gasparetto (2004), a Contabilidade é um importante provedor de informações para a avaliação do desempenho organizacional, isso porque essa avaliação é, de modo macro, a finalidade gerencial das informações que a Contabilidade fornece.

Ainda segundo o autor, os indicadores contábilfinanceiros, apesar de mostrarem apenas parcialmente o desempenho, têm revelado incontestável importância nessa tarefa. Mas não devem ser substituídos, e sim complementados com indicadores de outras áreas. Assim, ao invés de diminuir a importância dos indicadores contábil-financeiros na avaliação do desempenho organizacional deve-se complementá-los com outros indicadores mais amplos, de modo a obter uma análise global das empresas.

Nesse sentido, diversos são os esforços para consolidar o desempenho organizacional com foco contábil-financeiro num único indicador que seja capaz, então, de fornecer um ranking de performance. Um dos rankings mais conhecidos é o publicado anualmente pela Revista Exame em parceria com a Fundação Instituto de Pesquisas Contábeis, Atuariais e Financeiras (FIPECAFI), denominado Melhores e Maiores. Além desse ranking, diversos trabalhos procuram formar outros rankings de desempenho organizacional por meio da aplicação de ferramentas paramétricas e/ou não paramétricas. Entre esses trabalhos podem-se destacar os seguintes, tendo como base o foco do presente artigo: Babic e Plazibat (1998), Zhu (2000), Ceretta e Niederauer (2001), Yurdakul e Iç (2004), Santos e Casa Nova (2005), Macedo, Farias da Silva e Melo Santos (2006a e 2006b), Vilela, Nagano e Merlo (2007) e Onusic, Casa Nova e Almeida (2007) e Lyra (2008).

Assim sendo, o objetivo do presente trabalho é analisar o desempenho contábil-financeiro de empresas tomando como base os indicadores selecionados pela Melhores e Maiores (MM) da FIPECAFI/EXAME, para o ano de 2007, por meio da utilização das metodologias Análise Envoltória de Dados (DEA) e Análise Hierárquica (AHP). A intenção é comparar o ranking obtido pela metodologia da Melhores e Maiores (MM) com os rankings alcançados pelas metodologias da DEA e da AHP, com a finalidade de verificar a existência ou não de diferenças entre eles.

O propósito, então, concentra-se em explorar se metodologias tão diferentes são capazes de gerar rankings discrepantes, quando utilizam as mesmas variáveis e o mesmo peso/importância de cada vetor de desempenho. Isso será feito pela aplicação da DEA e da AHP para a formação de dois rankings de empresas do setor de Papel e Celulose e posterior comparação deles com o ranking publicado pela MM para esse mesmo setor.

Nesse contexto, tem-se, então, como problema de pesquisa, a seguinte questão central: Quais as diferenças existentes entre os rankings obtidos pelas metodologias da Melhores e Maiores (FIPECAFI/EXAME), da DEA e da AHP, na consolidação de índices para mensuração do desempenho organizacional com base em indicadores contábil-financeiros? Essa questão de pesquisa remete ainda a um questionamento secundário: Essas diferenças, se existentes, são significativas?

Os principais resultados mostram que as diferenças metodológicas produzem, par a par, uma baixa associação entre os rankings, mas quando o grau de concordância metodológica é mensurado há uma associação significativa entre os três. Ou seja, com base nesses resultados, pode-se afirmar que as diferenças metodológicas entre os rankings 
obtidos pela aplicação das três metodologias (MM, DEA e AHP), foram fracamente capturadas pela análise de correlação, porém não foram significativas para a medida de concordância utilizada.

Por fim, ressalta-se que o trabalho está estruturado nos seguintes itens, além desta introdução: fundamentação sobre desempenho organizacional com base em indicadores contábil-financeiros, metodologia, resultados, conclusão e referências.

\section{DESEMPENHO ORGANIZACIONAL COM FOCO CONTÁBIL- FINANCEIRO}

A mensuração de desempenho é definida por Neely (1998) como sendo o processo de quantificar a eficiência e a efetividade de ações passadas, mediante aquisição, coleta, classificação, análise, interpretação e disseminação dos dados apropriados, gerando informações importantes para que ações preventivas e/ou corretivas sejam tomadas.

Neely (1998) ressalta que as avaliações feitas em caráter relativo (benchmarking), ou seja, mensurando o desempenho da organização em relação, por exemplo, a seu ambiente competitivo, são geradoras de resultados potencialmente relevantes. Isso ocorre à medida que informam o posicionamento da empresa perante as demais, além de subsidiar a escolha de benchmarks (referências de desempenho).

Hendriksen e Van Breda (1999) enfatizam que a mensuração do desempenho pode ser feita sob a ótica de valores monetários, utilizando normalmente dados contábilfinanceiros, e/ou sob a ótica de informações não monetárias. A conjugação de informações de ambas as naturezas pode levar o sistema de avaliação do desempenho a uma condição diferenciada no que diz respeito a certas predições para subsidiar o processo decisório.

Ainda nessa linha de raciocínio, segundo Kaplan e Norton (1997), o uso de indicadores oriundos de práticas contábil-financeiras é importante, mas eles não conseguem traduzir todos os objetivos estratégicos das empresas. Por conta disso, muitos estudos vêm buscando integrar esses indicadores a outros não financeiros, formando, assim, uma avaliação focada em diversas naturezas do desempenho organizacional, visto que os gestores necessitam de índices que abranjam vários aspectos.

Gasparetto (2004) complementa ressaltando que os indicadores contábil-financeiros sozinhos são insuficientes, embora constituam um importante parâmetro (ou perspectiva) para a avaliação do desempenho organizacional, e a Contabilidade é responsável pelo provimento das informações necessárias para que esses indicadores sejam mensurados de forma confiável.

Apesar da indiscutível importância dos aspectos não financeiros na análise e na avaliação do desempenho organizacional, o que se percebe é que ainda se tem na análise contábil-financeira o foco principal de muitos estudos. De acordo com Omaki (2005), o uso de medidas de desempenho contábil-financeiro, a despeito das limitações, ainda é o procedimento mais comum, existindo inúmeros estudos comprovando sua validade, ao menos como um estimador razoável do desempenho organizacional.

Brito e Vasconcelos (2005) destacam que os indicadores contábil-financeiros acabam sendo usados, uma vez que estão disponíveis e são comparáveis, mesmo com a restrição de se ter mensurada uma limitada dimensão de desempenho. Os autores concluem ressaltando que o desempenho, ainda que seja apenas de caráter contábil-financeiro, tem uma natureza multidimensional ou multicriterial, e essas dimensões ou esses critérios podem ter objetivos conflitantes entre si, oferecendo dificuldades para a criação de um objetivo composto de desempenho.

Segundo Ching, Marques e Prado (2003) e Marques (2004) e Iudícibus (2008), a Contabilidade, por meio de suas demonstrações, pode ser considerada como uma das mais importantes linguagens dos negócios e uma das principais fontes de dados utilizadas para fins de avaliação de desempenho das empresas, porém sua utilização carece de um entendimento de suas limitações de uso no processo decisório.

Ainda para Ching, Marques e Prado (2003), Perez Jr. e Begalli (2002) e Matarazzo (2003), as demonstrações contábeis fornecem informações úteis e compreensíveis a respeito do passado para ajudar seus usuários a fazer previsões e tomar decisões relativas à situação econômicofinanceira futura do negócio. Isso é feito mediante análise contábil-financeira, quando se utiliza de uma série de índices calculados com base em relações entre contas ou grupos de 
contas das demonstrações contábeis.

Matarazzo (2003), Marques (2004) e Assaf Neto (2006) salientam, contudo, que a análise contábil-financeira, apesar de ser fonte de informações necessárias a qualquer abordagem de estudo de desempenho organizacional, tem como principal preocupação fornecer uma avaliação genérica sobre diferentes aspectos da empresa em análise, todos vistos sob a ótica econômico-financeira, sem descer a um nível maior de profundidade, que só seria alcançada com a inclusão de outras informações estratégicas da organização investigada.

Nesse sentido, Perez Jr. e Begalli (2002), Matarazzo (2003), Assaf Neto (2006) e Iudícibus (2008) distinguem uma questão que se torna interessante na análise contábilfinanceira: o que fazer com o conjunto de índices calculados no sentido de se obter uma visão do desempenho da entidade organizacional. O problema é que existem vários parâmetros de decisão e é bem possível que cada um deles leve a uma conclusão diferente e até mesmo conflitante em relação à situação econômico-financeira da empresa.

Sendo assim, segundo Pereira da Silva (2008) e Matarazzo (2003), é necessário, em benefício da própria análise contábil-financeira, que determinados instrumentos quantitativos sejam utilizados no sentido de auxiliar o uso dos resultados dessa análise no processo de tomada de decisão.

Para isso, é preciso aplicar metodologias que integrem os indicadores da análise contábil-financeira, de modo a organizar e a condensar a informação, haja vista a quantidade de indicadores e a diversidade de combinações possíveis para a análise de desempenho. Segundo Santos e Casa Nova (2005), o que está na base de aplicação dessas metodologias é o entendimento do desempenho como um fator multidimensional ou multicriterial, expresso mediante uma análise conjunta dos indicadores.

O emprego de Métodos Multicritério de Apoio à Decisão (MCDA) pode ser uma solução para esse tipo de problema. O Apoio Multicritério à Decisão (AMD) consiste, então, em um conjunto de métodos e técnicas para auxiliar ou apoiar a tomada de decisões, na presença de uma multiplicidade de critérios (GOMES, GONÇALEZ-ARAYA e CARIGNANO, 2004).

A resposta mais importante do uso desses métodos é a caracterização de uma medida de desempenho, que faz com que a decisão fique orientada por um único indicador construído com base em várias abordagens de desempenho diferentes. Vale ressaltar que isso facilita, e muito, o processo decisório, pois o analista, para concluir a respeito do desempenho da empresa, utiliza-se apenas da medida de performance resultado do uso de metodologias multicritério, ao invés de considerar vários índices.

Entre os métodos de Apoio Multicritério à Decisão (AMD), destacam-se a Análise Hierárquica (AHP - Analytic Hierarchy Process) e a Análise Envoltória de Dados (DEA), que constroem indicadores consolidados de desempenho, com base na comparação de vetores de performance. Sendo assim, vale salientar a importância do comportamento comparável das unidades sob análise em relação aos indicadores utilizados no processo de consolidação. Em outras palavras, é desejável que a amostra de empresas investigadas seja a mais homogênea possível em relação às características operacionais, contábeis, financeiras, mercadológicas, entre outras. Isso faz com que se isole a diferença entre os desempenhos para única e exclusivamente a performance em relação a cada vetor de desempenho utilizado na análise.

Segundo Gomes, Gonçalez-Araya e Carignano (2004), a AHP foi um dos primeiros métodos de análise multicritério e deve ser um dos mais usados no mundo. Vem sendo desenvolvido ao longo dos anos e hoje se pode dizer que integra uma família de métodos: Clássico, Multiplicativo, Referenciado e B-G.

De acordo com Anderson, Sweeney e Williams (2005), a AHP, desenvolvida por T. L. Saaty na década de 1970, é destinada a problemas de decisão multicriterial, a qual exige que o decisor faça julgamentos acerca da importância relativa de cada critério e depois especifique a preferência por cada alternativa, usando cada critério.

Nesse método, acrescentam Gomes, Gonçalez-Araya e Carignano (2004), a análise é dividida em níveis hierárquicos, ou seja, para a análise de cada alternativa são utilizados critérios/atributos divididos em vários graus hierárquicos. Convém ressaltar que os critérios em um mesmo nível devem ser homogêneos, e não redundantes.

De acordo com Gomes (2007), a montagem dessa estrutura hierárquica de análise é, geralmente, a parte mais difícil do método, constituindo-se, assim, no cerne 
da estruturação da AHP. Depois de definida a estrutura hierárquica, salienta Costa (2002), é necessária a definição de prioridades, ocasião em que o decisor ajusta suas preferências, comparando pares à luz de cada um dos critérios (julgamentos paritários).

Ainda segundo Gomes (2007), escolher um método de agregação de critérios, como a AHP, é equivalente a optar por um tipo de compensação entre os atributos. Ou seja, contrabalancear a desvantagem de um critério em relação a uma vantagem de outro. Assim, os métodos compensatórios, de modo geral, podem favorecer alternativas não balanceadas (ótimas em algum critério, mas sofrível em outros), já que buscam a compensação. Têm, porém, a vantagem de trabalhar com a ideia de agregar todas as informações numa grande síntese, que na AHP é decorrente da hierarquização dos critérios/atributos e das alternativas diante deles, até a obtenção da lista final, que contém os pesos globais das diversas alternativas.

Shimizu (2001) diz que a AHP tem sido um dos métodos mais comentados e aplicados na prática das decisões com múltiplos critérios, envolvendo complexidade e subjetividade. Para se ter uma ideia da abrangência e da aplicabilidade desse método, numa busca por bases de artigos científicos utilizando como palavra-chave AHP, obteve-se a seguinte resposta: Emerald (533 artigos); Scielo (17 artigos) e Science Direct (todas as ciências = 6155 artigos; business, management and accounting $=422$ artigos; economics, econometrics and finance $=262$ artigos). Em sua maioria, esses artigos representam aplicações do método e de algumas derivações dele, em problemas decisórios que envolvem escolha de tecnologia; seleção de fornecedores; avaliação do desempenho de pessoas (trabalhadores, alunos, entre outras); análise e seleção de investimentos; decisões de manutenção preventiva; escolha de software e hardware; problemas de alocação de recursos; desempenho em/de cadeias de suprimento; decisões de localização; consolidação das perspectivas do BSC; negociação e resolução de conflitos; avaliação de qualidade de serviços; desempenho ambiental; decisões em logística; alocação de custos (sistema tradicional e $\mathrm{ABC}$ ); desempenho de empresas; seleção de pessoas; decisões de terceirização; priorização de atividades; escolha de sistemas de produção; decisões de compra e consumo; desempenho de nações, entre outros.
Como se pode observar, algumas dessas aplicações são mais pertinentes à área contábil-financeira. Alguns artigos, tais como os de Frezatti (2007) e de Frezatti et al. (2007), utilizam a AHP como ferramenta de suporte à análise conduzida nos trabalhos. Outros, como os de Matos e Moura (2003) e de Baía e Machado (2006), utilizam a AHP como suporte a decisões de análise do custo de capital.

Uma das aplicações mais pertinentes de AHP é como método de apoio ao ABC (Activity Based Costing). Entre os trabalhos que integram ABC/AHP estão os de Ribeiro e Costa (1999), Pamplona (1999), Galvão, Cogan e Santos (2001), Campos e Ribeiro (2002), Ribeiro e Campos (2005) e Abreu e Campos (2007).

Mais voltados para a questão de análise do desempenho organizacional, alguns trabalhos integram diferentes perspectivas de performance utilizando a AHP, entre os quais destacam-se Sanches da Silva, Bornia e Pamplona (2006), Rafaeli e Muller (2007) e Albano (2008). Além desses, outros trabalhos, como os de Kimura et al. (1999) e de Kimura e Suen (2003), utilizam as perspectivas do BSC (Balanced Scorecard) para serem consolidadas pela AHP.

Tratando mais especificamente de desempenho organizacional com foco contábil-financeiro, tem-se os trabalhos de Babic e Plazibat (1998), de Lyra (2008) e de Yurdakul e Iç (2004), este último com objetivo específico de análise de crédito.

Já a Análise Envoltória de Dados (DEA), segundo Lins e Meza (2000), apresenta-se como método que fornece uma medida de desempenho capaz de comparar a eficiência de várias unidades similares mediante a consideração explícita do uso de suas múltiplas entradas para a produção de múltiplas saídas. Dessa forma, essa metodologia faz com que a decisão fique orientada por um único indicador, construído a partir de várias abordagens de desempenho diferentes.

Um dos maiores benefícios do uso da DEA, de acordo com Macedo, Farias da Silva e Melo Santos (2006a), é o conjunto de unidades de referência que pode ser usado como benchmarking na melhoria do desempenho das unidades menos eficientes. Esses benchmarks indicam o que precisa ser modificado nos inputs e outputs e como melhorá-los para transformar unidades ineficientes em eficientes.

A Análise Envoltória de Dados (DEA), segundo Zhu 
(2000), representa uma das mais adequadas ferramentas para avaliar a eficiência, em comparação com ferramentas convencionais. Os resultados da DEA são mais detalhados do que os obtidos em outras abordagens, servindo melhor ao embasamento de recomendações de natureza gerencial.

Apesar de originalmente ser uma metodologia proposta para um ambiente de produção (transformação de insumos em produtos), salientam Macedo, Farias da Silva e Melo Santos (2006b), a DEA pode ser empregada como um método multicritério quando se utilizam indicadores do tipo "quanto menor melhor" no lugar dos inputs (a exemplo de risco, custo, endividamento), e do tipo "quanto maior melhor" no lugar dos outputs (lucratividade, retorno, liquidez, entre outros). Isso transforma a DEA em um método de Apoio Multicritério à Decisão (AMD), aplicado com o intuito de consolidar várias perspectivas (critérios) de desempenho diferentes.

ADEAjá foi utilizada em vários trabalhos para consolidar medidas de desempenho organizacional, especialmente as de natureza contábil-financeira, entre os quais se destacam: Zhu (2000), Ceretta e Niederauer (2001), Santos e Casa Nova (2005), Macedo, Farias da Silva e Melo Santos (2006a e 2006b), Vilela, Nagano e Merlo (2007) e Onusic, Casa Nova e Almeida (2007).

Este artigo se insere exatamente nesse contexto de uso da AHP e da DEA como ferramentas de consolidação de índices contábil-financeiros para análise do desempenho organizacional. Porém o foco estará menos em explorar o desempenho obtido com o uso dessas ferramentas, ponto central da maioria dos artigos listados anteriormente, e mais em analisar comparativamente os dois instrumentos metodológicos e mais a metodologia utilizada pela MM na formação de rankings de desempenho organizacional centrado no aspecto contábil-financeiro.

\section{Metodologia}

\subsection{Aspetos Gerais}

Esta pesquisa pode ser caracterizada, de acordo com Vergara (2009), como descritiva e quantitativa, pois se procura, por meio da aplicação das Metodologias Análise Hierárquica (AHP) e Análise Envoltória de Dados (DEA) às informações das empresas do setor de Papel e Celulose no Brasil sob investigação, expor características a respeito da performance contábil-financeira desses empreendimentos com o objetivo de comparar os resultados com os obtidos pela metodologia da Melhores e Maiores (FIPECAFI/ EXAME).

A escolha do setor de Papel e Celulose se deu única e exclusivamente em função da homogeneidade das empresas, característica extremamente importante para metodologias tais como DEA e AHP, pois seus rankings são obtidos por comparações entre as organizações. Logo, a homogeneidade é um elemento crucial para dar representatividade à análise de desempenho conduzida pelo presente estudo.

O processo de amostragem é não probabilístico, pois se baseia num universo naturalmente restrito, já que as empresas foram escolhidas pela base de dados Melhores e Maiores da Exame-FIPECAFI. Para fazer parte da amostra final, as empresas precisariam estar listadas entre as dez melhores do setor na base de dados do ano de 2007 (edição 2008).

Os dados utilizados são secundários e extraídos da base da Melhores e Maiores da Exame-FIPECAFI, para o ano de 2007. No total tinha-se seis variáveis, utilizadas para formar o ranking MM, que foram consideradas para fins de análise: crescimento das vendas, participação de mercado, RSPLajustada, LC, investimento no imobilizado e riqueza criada por empregado. A escolha dessas variáveis se justifica pelo fato de se ter como objetivo a comparação entre os rankings. Sendo assim, apenas a utilização das mesmas variáveis torná-los-ia comparáveis. Cabe ressaltar que também foram utilizados os mesmos pesos definidos pela base MM. O Quadro 1, a seguir, mostra as variáveis utilizadas e seus respectivos pesos, para cada organização analisada: 
Quadro 1 - Informações Utilizadas na Análise

\begin{tabular}{|c|c|c|c|c|c|c|c|}
\hline Código MM & Nome Fantasia & $\begin{array}{c}\text { Partic. de Mercado (PM) } \\
\text { Peso }=15 \%\end{array}$ & $\begin{array}{c}\text { Cresc das Vendas (CV) } \\
\text { Peso }=10 \%\end{array}$ & \begin{tabular}{|c|} 
RSPL Aj. \\
Peso $=25 \%$
\end{tabular} & \begin{tabular}{|c|} 
Riq Criada por empr (RC) \\
Peso $=15 \%$
\end{tabular} & \begin{tabular}{|c|} 
LC \\
Peso $=20 \%$
\end{tabular} & $\begin{array}{c}\text { Invests no imob. (IMB) } \\
\text { Peso }=15 \%\end{array}$ \\
\hline 408 & Aracruz & 14.4157 & 28.6540 & 14.3340 & 142.9460 & 1.9342 & 21.7660 \\
\hline 1066 & Celulose Irani & 2.4734 & 35.0860 & 21.4210 & 39.1930 & 1.1769 & 56.0800 \\
\hline 153 & Cenibra & 7.2663 & 38.3170 & 24.4780 & 155.5820 & 1.3291 & 9.5430 \\
\hline 346 & International Paper-SP & 11.1682 & 90.9540 & 0.5060 & 119.2780 & 3.5877 & 76.6940 \\
\hline 137 & Klabin & 19.2578 & 24.0240 & 19.6050 & 71.6550 & 2.3153 & 63.3580 \\
\hline 1376 & Mili & 2.0547 & 38.0970 & 15.0790 & 50.0580 & 1.9048 & 47.2100 \\
\hline 4057 & Pisa & 1.6726 & 4.5900 & 14.9860 & 149.6000 & 0.8888 & 70.8950 \\
\hline 47 & Santher & 4.8621 & 28.8500 & 27.3950 & 85.1710 & 0.9361 & 10.1470 \\
\hline 312 & Suzano & 21.7486 & 40.9170 & 13.2080 & 188.3680 & 2.2211 & 21.9980 \\
\hline 258 & $\mathrm{VCP}$ & 15.0805 & 9.5770 & 8.6880 & 225.4480 & 1.3138 & 10.7280 \\
\hline
\end{tabular}

Fonte: Elaborado pelos autores

Com base nas informações do Quadro 1, procedeu-se à análise de desempenho contábil-financeira das empresas de Papel e Celulose, proposta por este estudo. Empregando os mesmos indicadores utilizados pela MM, bem como seus respectivos pesos, aplicaram-se as metodologias de análise e avaliação do desempenho multicriterial, no caso, a AHP e a DEA.

Em uma análise de desempenho, ressaltam Gomes, Gomes e Almeida (2002), pode-se utilizar uma análise monocritério ou uma multicritério. NaAnálise Monocritério, que neste caso pode ser expressa por cada um dos índices contábil-financeiros listados, pode-se valorar cada ação potencial sobre um único eixo de significância, ou seja, uma unidade comum. Já na Análise Multicritério tem-se um novo paradigma que reconhece a heterogeneidade das dimensões de desempenho, pela utilização de uma família de múltiplos critérios, escolhidos segundo exigências técnicas, inteligibilidade, aceitação como base do trabalho e facilidade de utilização como instrumento de análise.

Neste artigo, para consolidar o desempenho contábilfinanceiro, utilizam-se duas ferramentas de Análise Multicritério: o Método de Análise Hierárquica (AHP) e a Análise Envoltória de Dados (DEA), com as quais é possível avaliar o desempenho de cada empresa do setor de Papel e Celulose de modo multicriterial, considerando de maneira integrada todos os vetores de desempenho apresentados em cada modelo monocriterial.

\subsection{AHP}

Como dito anteriormente, a AHPé um método de Apoio Multicritério à Decisão (AMD), que, conforme Anderson,
Sweeney e Williams (2005) e Costa (2002), tem como resposta um ranking das alternativas sob análise, priorizadas segundo a preferência geral do decisor ante todos os critérios. Isso é feito, de acordo com Gomes, Gonçalez-Araya e Carignano (2004), por meio de comparações dos critérios, par a par, e depois das alternativas em relação a cada critério, criando-se várias matrizes de decisão quadrada, em que a cada critério e a cada alternativa associa-se um valor de prioridade sobre as outras analisadas, a partir de uma escala fundamental de preferências.

Ainda segundo Anderson, Sweeney e Williams (2005), a base da AHP é, portanto, a análise comparativa, par a par, tanto dos critérios quanto das alternativas, para definir preferências (hierarquizar). Para isso, complementam os autores, é necessário estabelecer quão importante é cada critério em relação a cada um dos outros critérios e, dentro de cada critério, quão importante é cada alternativa em relação a todas as outras alternativas. Isso é feito por meio da construção de uma matriz quadrada de decisão, em que os critérios e as alternativas podem ser comparados aos pares, seguindo a escala de comparação de Saaty, descrita a seguir, no Quadro 2.

Pode-se perceber, segundo Gomes, Gonçalez-Araya e Carignano (2004) e Anderson, Sweeney e Williams (2005), que a escala define apenas o que é mais importante, e quão mais importante. Por exemplo, no caso de um critério i ter importância grande ou essencial em relação a j, será atribuído o valor 5 da escala para a comparação de i em relação a j (linha i e coluna j da matriz de decisão). Já na comparação de j em relação a i (linha j e coluna i da matriz de decisão) será atribuído o inverso desse valor, ou seja, 1/5. Para o menos importante, pois, a comparação assume o valor inverso ou recíproco da escala original. 
No caso do presente trabalho não foi feita a priorização em relação à comparação dos critérios para determinar seus graus de importância porque, para os critérios, esses graus representam seus pesos no modelo. Portanto, como se utilizam os pesos da base MM, não se faz essa etapa do método AHP.
Para construir o indicador final, que revela a hierarquia das alternativas ante todos os critérios, é necessário fazer para cada alternativa o somatório dos produtos entre o valor de seu índice dentro de cada critério e o peso de cada critério. Ou seja, para cada alternativa é feito o somatório dos produtos entre a importância do critério e o índice da importância no critério. $\mathrm{O}$ resultado dessa conta mostra um índice de prioridade geral para cada uma das alternativas, sendo, então, possível hierarquizá-las.

Quadro 2 - Escala de Comparações de Saaty

De acordo com Anderson,

\begin{tabular}{|c|c|c|}
\hline $\begin{array}{l}\text { Intensidade de } \\
\text { Importância }\end{array}$ & Definição & Explicação \\
\hline 1 & Mesma Importância & $\begin{array}{l}\text { As duas atividades contribuem igualmente para } 0 \\
\text { objetivo. }\end{array}$ \\
\hline 3 & Importância pequena de uma sobre a outra & $\begin{array}{l}\text { A experiência e } 0 \text { julgamento favorecem } \\
\text { levemente uma atividade em relação a outra. }\end{array}$ \\
\hline 5 & Importância grande ou essencial & 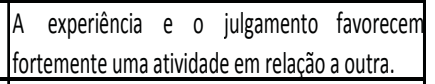 \\
\hline 7 & Importância muito grande ou demonstrada & $\begin{array}{l}\text { Uma atividade é muito fortementefavorecida em } \\
\text { relação a outra; sua dominação de importância é } \\
\text { demonstrada na prática. }\end{array}$ \\
\hline 9 & Importância absoluta & $\begin{array}{l}\text { A evidência favorece uma atividade em relação a } \\
\text { outra com o mais alto grau de certeza. }\end{array}$ \\
\hline $2,4,6,8$ & Valores intermediários entre os valores adjacentes & $\begin{array}{l}\text { Quando se procura uma condição de } \\
\text { compromisso entre duas definições. }\end{array}$ \\
\hline $\begin{array}{l}\text { Recíprocos dos } \\
\text { valores acima de } \\
\text { zero }\end{array}$ & $\begin{array}{l}\text { Se a atividade i recebe uma das designações dif } \\
\text { acima de zero, quando comparada com a atividade } \\
\text { jtem o valor recíproco quando comparada com i }\end{array}$ & UUma designação razoável. \\
\hline Racionais & Razões resultantes da escala & $\begin{array}{l}\text { Se a consistência tiver de ser forçada para obtel } \\
\text { valores numéricos } n \text {, para completar a matriz. }\end{array}$ \\
\hline
\end{tabular}

Fonte: Saaty (1991). Sweeney e Williams (2005) e com Costa (2002), para todas as matrizes de comparação (entre critérios e entre alternativas para cada critério) é necessário testar a consistência da análise e do modelo construído. Segundo Saaty (1991) o teste de consistência tem de ser de tal ordem que se possa testar ao mesmo tempo não só a violação de transitividade (A é preferível a B; B é preferível a C; logo, A tem de ser preferível a C) como também a de proporcionalidade (A é duas vezes preferível a B; B é três vezes preferível a $\mathrm{C}$; logo, $\mathrm{A}$ tem de ser seis vezes preferível a $\mathrm{C}$ ).

De acordo com Anderson, Sweeney e Williams (2005) e com Gomes, Gonçalez-Araya e Carignano (2004), para priorizar cada alternativa dentro de cada critério, a partir da matriz de comparação, é utilizado um método de sintetização das preferências do AHP. Primeiramente, somam-se os valores de cada coluna da matriz, depois se constrói uma nova matriz dividindo cada valor da matriz original pelo somatório de sua coluna, efetuando o que se denomina normalização da matriz. Em seguida é calculada a média de cada linha da matriz normalizada para se achar a priorização/ hierarquização das alternativas ante um determinado critério. Cabe ressaltar que a soma dos índices de priorização das alternativas dentro de um critério deve ser igual a 1,00. O mesmo procedimento é repetido para todos os critérios, de modo a obter uma hierarquização das alternativas ante cada critério.

Conforme Saaty (1991), para verificar a consistência da análise são necessárias algumas operações entre matrizes. Primeiramente multiplica-se a matriz de comparação pelo vetor de pesos (resultado hierarquizado para os critérios ou alternativas para cada critério ou vetor de priorização dos critérios ou alternativas), e tem-se, assim, um novo vetor. Divide-se a primeira componente desse vetor pela primeira componente do vetor de pesos, a segunda componente desse vetor pela segunda componente do vetor de pesos e assim por diante, até se obter um novo vetor. A média dos elementos deste último vetor será o $\lambda \max$. O quociente entre $(\lambda \max -$ n) e (n -1) é o que Anderson, Sweeney e Williams (2005) denominam índice de consistência (IC), o qual, de acordo com Saaty (1991), tenderá a zero quanto mais consistente for o resultado. 
Para analisar a consistência, de acordo com Gomes, Gonçalez-Araya e Carignano (2004), utiliza-se um indicador denominado razão de consistência (RC), obtido pela divisão do IC por um índice aleatório, calculado para matrizes quadradas de ordem n pelo Laboratório Nacional de Oak Ridge, nos EUA, denominado índice randômico (IR). Quando $\mathrm{n}>4$, o valor aceitável para a razão de consistência é $\mathrm{RC} \leq 0,10$. Os valores do IR, para matrizes quadradas com $\mathrm{n}=10$ (que é o caso das matrizes de comparação entre as alternativas dentro de cada critério neste trabalho), de acordo com Saaty (1991), tende a 1,49, que será o valor considerado nesta análise. Os resultados dos testes de consistência mostram que todas as razões de consistência (RC) estavam abaixo do limite $(\mathrm{RC} \leq 0,10)$.

\subsection{DEA}

De acordo com Charnes et al. (1994) e com Coelli, Rao e Baltese (1998), a Análise Envoltória de Dados (DEA) mostra quão eficiente é uma empresa, no tratamento de seus inputs e outputs, em relação às outras, numa análise a qual fornece um indicador que varia de 0 a 1 ou de $0 \%$ a $100 \%$. Somente as empresas que obtêm índice de eficiência igual a um é que fazem parte da fronteira eficiente.

Destaca-se que a modelagem não se limita à avaliação de desempenho, fornecendo também os pontos ótimos que as organizações deveriam atingir em relação aos parâmetros determinados. Essa análise recebe o nome de benchmarking, caracterizando-se por procurar propor, com base no que as unidades eficientes já conseguem atingir, valores ideais para os indicadores das unidades tidas como não eficientes.

Em termos práticos, de acordo com Santos e Casa Nova (2005), o modelo procura identificar a eficiência de uma empresa do setor elétrico, comparando-a com os melhores desempenhos observados em cada ano, por meio da resolução do seguinte problema de programação linear (PPL):

$$
\begin{array}{ll}
\operatorname{Max} E_{c}= & \sum_{j=1}^{s} u_{j} y_{\dot{\mathcal{x}}} \\
\text { S.a.: } \quad & \sum_{i=1}^{m} v_{i} x_{\dot{c}}=1 \quad \text { Orientação input } \\
& \sum_{j=1}^{s} u_{j} y_{k}-\sum_{i=1}^{m} v_{i} x_{k} \leq 0, k=1,2, \ldots, c, \ldots, n \\
& u_{j}, v_{i} \geq 0, \forall x, y .
\end{array}
$$

Nesse modelo, com orientação input (DEA-CRS-I), c é a unidade (DMU - Decision Making Units) que está sendo avaliada. O problema acima envolve a procura de valores para uj e vi, que são os pesos (importância relativa de cada variável/critério), de modo que maximize a soma ponderada dos outputs (yj) da DMU em estudo, sujeita às restrições de que a soma ponderada dos inputs (xi) dessa mesma DMU seja igual a um, e a diferença entre a soma ponderada dos outputs (yj) e a soma ponderada dos inputs (xi) seja menor ou igual a zero, para todas as DMUs. Esta última restrição faz com que nenhuma unidade excederá $100 \%$ de eficiência quando o mesmo conjunto de coeficientes de entrada e saída (os vários uj e vi) for aplicado a todas as outras unidades que estão sendo comparadas.

Uma das características da DEA é ser uma medida relativa de desempenho, ou seja, os scores verificados em determinada função somente valem para a amostra em estudo. Caso haja alteração na amostra com inclusão ou exclusão de novas DMUs ou alteração nas variáveis, tornase necessário calcular novos scores de eficiência. Em síntese, os resultados de eficiência de determinada amostra não são comparáveis aos resultados de amostras diferentes e nem da população.

Convém ressaltar que essa é uma característica não paramétrica da metodologia DEA, em que os parâmetros de desempenho são reais e estabelecidos dentro da amostra, levando em conta as variáveis pesquisadas. Salientase, ainda, a impossibilidade de trabalhar com variáveis negativas, como um problema apresentado pela modelagem DEA. Por conta disso, procedeu-se a ajustes pela soma de uma constante para a variável crescimento das vendas, no início da análise. Além disso, acrescenta-se ao PPL acima restrições aos pesos dos outputs e inputs, de forma a manter os mesmos pesos utilizados na formação do ranking pela MM.

\subsection{AnÁLISE COMPARATIVA dos RANKINGS}

Para comparar as três metodologias, faz-se necessário explorar, em linhas gerais, como é formado o ranking de desempenho organizacional com base em indicadores contábil-financeiros da MM:

- $\quad$ Em cada indicador, as empresas de um mesmo 
setor são ordenadas de maneira decrescente em relação ao seu desempenho. Depois disso é atribuída uma pontuação, em que o primeiro colocado fica com dez pontos até o décimo colocado, que fica com um ponto.

- Depois dos pontos atribuídos a cada empresa, a pontuação final é obtida pela soma ponderada dos pontos, em que cada indicador possui um peso, conforme descrito no Quadro 1.

- $\quad$ De posse da pontuação das empresas, é possível estabelecer um ranking do desempenho organizacional com foco contábil-financeiro para cada setor analisado.

Cabe destacar aqui que, para fins de comparação, foram desconsiderados os pontos atribuídos pela MM à participação das empresas nos guias de Sustentabilidade e Melhores Empresas para Trabalhar.

Por fim, para poder comparar os três rankings (MM, AHP e DEA), procedeu-se ao cálculo de duas medidas não paramétricas de correlação e outra de concordância, todas em nível de significância de $10 \%$. Para medir a correlação entre os rankings, executaram-se as análises de correlação de posto-ordem de Spearman e de Kendall, com auxílio do software SPSS 16.0. Já para medir o grau de concordância entre os rankings, calculou-se o coeficiente de concordância W de Kendall, com auxílio do Excel.

Segundo Siegel e Castellan Jr. (2006), as medidas não paramétricas de correlação de posto-ordem de Spearman e de Kendall requerem que as variáveis sejam medidas pelo menos em uma escala ordinal, de modo que os objetos em estudo possam ser colocados em postos de duas séries ordenadas. Logo, as comparações foram feitas aos pares: MM x AHP, MM x DEA e AHP x DEA.

Ainda segundo os autores, a medida de concordância $\mathrm{W}$ de Kendall mede o grau de associação entre $\mathrm{K}$ conjuntos de postos de $\mathrm{N}$ objetos. $\mathrm{O}$ coeficiente de concordância seria, então, um índice da divergência entre a verdadeira concordância mostrada nos dados e a concordância perfeita.

\section{ApresentaÇão e anÁlise dos resultados}

A Tabela 1 mostra os resultados, em termos de indicadores de desempenho contábil-financeiro, em relação às aplicações da AHP e da DEA.
Tabela 1 - Indicadores AHP e DEA do Desempenho Contábil-Financeiro

\begin{tabular}{|c|l|c|c|}
\hline Código MM & \multicolumn{1}{|c|}{ Nome Fantasia } & Índice AHP & Índice DEA \\
\hline 408 & Aracruz & $8.12 \%$ & $72.03 \%$ \\
\hline 1066 & Celulose Irani & $7.89 \%$ & $21.59 \%$ \\
\hline 153 & Cenibra & $9.37 \%$ & $45.01 \%$ \\
\hline 346 & International Paper-SP & $15.79 \%$ & $4.03 \%$ \\
\hline 137 & Klabin & $10.94 \%$ & $100.00 \%$ \\
\hline 1376 & Mili & $7.52 \%$ & $25.94 \%$ \\
\hline 4057 & Pisa & $7.23 \%$ & $7.42 \%$ \\
\hline 47 & Santher & $11.01 \%$ & $19.83 \%$ \\
\hline 312 & Suzano & $12.80 \%$ & $97.23 \%$ \\
\hline 258 & VCP & $9.32 \%$ & $22.69 \%$ \\
\hline
\end{tabular}

Fonte: Elaboração dos autores.

Como dito anteriormente, o resultado da AHP é um indicador cuja soma totaliza $100 \%$. Percebe-se que, em relação à AHP, a melhor empresa foi a International PaperSP, seguida pela Suzano, pela Santher e pela Klabin. As piores colocações ficaram com a Pisa, a Mili e a Celulose Irani.

Quanto à DEA, o indicador de cada empresa pode variar de $0 \%$ a $100 \%$, e somente as organizações com valor igual a $100 \%$ podem ser consideradas eficientes. Nesse caso, observa-se que a melhor empresa foi a Klabin, seguida pela Suzano. As piores foram a International Paper-SP e a Pisa, ambas com desempenho muito inferior ao das demais.

A comparação entre os rankings pode ser feita primeiramente, de maneira visual, pela análise da Tabela 2, em que são apresentadas as posições de cada empresa em cada ranking.

Tabela 2 - Rankings MM, AHP e DEA

\begin{tabular}{|c|l|c|c|c|}
\hline Código MM & \multicolumn{1}{|c|}{ Nome Fantasia } & Ranking MM & Ranking DEA & Ranking AHP \\
\hline 312 & Suzano & 1 & 2 & 2 \\
\hline 346 & International Paper-SP & 2 & 10 & 1 \\
\hline 137 & Klabin & 3 & 1 & 4 \\
\hline 153 & Cenibra & 4 & 4 & 5 \\
\hline 408 & Aracruz & 5 & 3 & 7 \\
\hline 1376 & Mili & 6 & 5 & 9 \\
\hline 4057 & Pisa & 7 & 9 & 10 \\
\hline 1066 & Celulose Irani & 8 & 7 & 8 \\
\hline 47 & Santher & 9 & 8 & 3 \\
\hline 258 & VCP & 10 & 6 & 6 \\
\hline
\end{tabular}

Fonte: Elaboração dos autores. 
Percebe-se, pela análise inicial da Tabela 2, que a primeira colocada no ranking MM é a segunda melhor empresa, tanto pela AHP quanto pela DEA. Além disso, a segunda melhor da MM é a melhor pela AHP, e a terceira é a melhor pela DEA. Os maiores problemas são observados pelo fato de a segunda melhor empresa pela MM ser a pior pela DEA e pelo fato de a penúltima empresa pela MM ser a terceira melhor pela AHP. No restante não há variações visuais relevantes.

Porém, para concluir a respeito da significância dessas alterações entre os rankings, procedeu-se à análise de medidas de associação: grau de correlação de Spearman e de Kendall e grau de concordância W de Kendall. Primeiramente, os resultados das correlações, bem como seus níveis de significância, podem ser vistos na Tabela 3 , a seguir:

Tabela 3 - Correlações de Spearman e de Kendall para os Rankings MM, AHP e DEA

\begin{tabular}{|c|c|c|c|c|c|}
\hline \multicolumn{3}{|c|}{ SPEARMAN } & \multicolumn{3}{c|}{ KENDALL } \\
\hline Rankings & AHP & DEA & Rankings & AHP & DEA \\
\hline \multirow{2}{*}{ MM } & 0.527 & 0.418 & \multirow{2}{*}{ MM } & 0.422 & 0.333 \\
\cline { 2 - 3 } \cline { 5 - 6 } & $(0.117)$ & $(0.229)$ & & $(0.089)$ & $(0.180)$ \\
\hline \multirow{2}{*}{ AHP } & & 0.091 & \multirow{2}{*}{ AHP } & & 0.111 \\
\cline { 2 - 3 } \cline { 5 - 6 } & & $(0.803)$ & & $(0.655)$ \\
\hline
\end{tabular}

Fonte: Elaboração dos autores.

Observa-se, pela análise da Tabela 3, que os rankings possuem medidas de associação não significativas, de maneira geral a $10 \%$. A única exceção foi a correlação entre os rankings AHP e MM, pela medida de Kendall, que se mostrou fraca, mas significativa. Cabe ressaltar que a significância pela medida de Spearman, para essa mesma relação, foi apenas um pouco superior a $10 \%$.

No entanto, além das medidas de correlação, verificouse o grau de concordância entre os três rankings pela medida de associação W de Kendall, que procura examinar, mesmo que, de par a par, a correlação não seja alta, se existe - em geral - um certo grau de concordância entre os rankings.
Ou seja, procura responder se existe associação significativa numa observação conjunta dos três rankings.

Os resultados para o $\mathrm{W}$ de Kendall mostram que existe associação significativa para os três rankings ao nível de $10 \%$ de significância. Isso porque o valor do W de Kendall para o nível de concordância entre os três rankings foi de 0,5636 , que gera um valor calculado para o qui-quadrado de 15,22 , maior que o valor tabelado para $\mathrm{gl}=9$ de 14,68 . Logo, pode-se rejeitar $\mathrm{H} 0$ e concluir que há um nível de concordância significativo entre os três rankings.

Assim, constata-se que apesar de, par a par, as diferenças metodológicas produzirem uma baixa associação entre os rankings, eles mostram uma associação significativa quando o grau de concordância entre os três é mensurado.

\section{Conclusão e Considerações Finais}

Este trabalho teve como propósito analisar comparativamente o desempenho contábil-financeiro de empresas do setor de Papel e Celulose, aplicando os recursos metodológicos AHP e DEA aos mesmos indicadores considerados pela metodologia da MM para formar seu ranking. Levaram-se em conta, ainda, os mesmos pesos aplicados pela MM a esses indicadores, haja vista o objetivo secundário que se tinha de comparar a posição das empresas nos três rankings.

Percebe-se que existem diferenças entre os rankings, pois as melhores e as piores empresas nos três rankings não são totalmente coincidentes. Para testar, porém, a existência ou não de diferença significativa entre eles, obtiveram-se algumas medidas de associação.

No que diz respeito às análises de correlação (Spearman e Kendall), observa-se que, de maneira geral, podese rejeitar a hipótese de que as três metodologias gerem rankings correlacionados. Isso quer dizer que cada metodologia, baseada inclusive nos mesmos indicadores e nos mesmos pesos, é capaz de gerar um ranking diferente para as mesmas empresas.

Já quando se utiliza o grau de concordância mensurado pelo W de Kendall, nota-se que os três rankings possuem associação significativa ao nível de $10 \%$. Ou seja, pode-se aceitar a hipótese de concordância significativa entre os rankings, apesar das diferenças observadas e da 
não correlação entre cada par de ordenação.

Vê-se que essas respostas são conflitantes, o que denota que as diferenças metodológicas foram capturadas pela análise de correlação, porém não foram significativas para a medida de concordância utilizada.

De maneira geral, as três ferramentas de ordenação são diferentes do ponto de vista metodológico. Isso se deve ao fato de que o ranking MM ordena exclusivamente em função da posição de cada empresa em relação a cada variável. Ou seja, diferentemente da DEA e da AHP, o ranking MM não considera as diferenças de magnitude das variáveis, mas apenas sua ordenação. Já os rankings DEA e AHP, apesar de levarem em conta as diferenças nos valores das variáveis para formar o ranking, fazem isso de maneira diferente, já que a DEA julga a diferença em termos relativos e a AHP a interpreta em termos de grupos formados em relação à escala fundamental de Saaty.

Apesar dessas diferenças e de se observar pouca aderência par a par entre as metodologias, uma análise conjunta das três mostra existir uma consistência significativa entre os rankings formados por elas, o que permite concluir que não existem diferenças significativas nos resultados embora haja diferenças importantes nas ferramentas utilizadas.

Por fim, cabe salientar que a metodologia da MM possui a grande vantagem de ser de fácil implementação, todavia se mostra frágil em gerar outras respostas que não seja especificamente o ranking de desempenho. Já a DEA e a AHP têm uma implementação mais complicada, apesar da existência de softwares que facilitam esse processo. São metodologias que apresentam limitações, restrições e características específicas de aplicação, mas que oferecem outros benefícios além do ranking. $\mathrm{Na}$ AHP, por exemplo, existe a possibilidade de se discutir melhorias necessárias para atingir indicadores-alvo e na DEA a chance de melhorar aspectos competitivos utilizando os melhores desempenhos como benchmarks.

Sendo assim, os pesquisadores precisam estar atentos ao que as metodologias podem particularmente oferecer e quais os custos para aplicá-las, de modo a analisar a relação custo $\mathrm{x}$ benefício no processo de escolha por uma delas. Além disso, é necessário estar ciente das limitações existentes quando se procura consolidar múltiplas perspectivas em um único indicador.
Este trabalho pode ter continuidade pelo estudo dos índices de desempenho contábil-financeiro gerados pelas três metodologias, procurando compará-los por testes de diferença de médias e regressões que apontem os fatores mais significativos para a formação desses indicadores.

\section{REFERÊNCIAS}

ABREU, A. O.; CAMPOS, R. O Método ABC/AHP aplicado em uma Indústria de Serviços. In: ENCONTRO NACIONAL DE ENGENHARIA DE PRODUÇÃO, 27. 2007, Foz do Iguaçu. Anais do XXVII ENEGEP. Foz do Iguaçu: ABEPRO, 2007. CD-ROM.

ALBANO, F. M. Desenvolvimento de um Modelo de Avaliação Global de Desempenho. 2008. 122 f. Dissertação (Mestrado em Engenharia de Produção) - Escola de Engenharia, Universidade Federal do Rio Grande do Sul, Rio Grande do Sul.

ANDERSON, D. R.; SWEENEY, D. J.; WILLIAMS, T. A. An Introduction to Management Science: quantitative approaches to decision making. 11 ed. Ohio: Thomson/South-Western College, 2005.

ASSAF NETO, A. Estrutura e Análise de Balanços: um enfoque econômico-financeiro. 8 ed. São Paulo: Atlas, 2006 .

BABIC, Z.; PLAZIBAT, N. Ranking of enterprises based on multicriterial analysis. International Journal of Production Economics. v. 56-57, p. 29-35, 1998. <http:// dx.doi.org/10.1016/S0925-5273(97)00133-3>

BAÍA, G. C.; MACHADO, M. A. V. Estudo da influência dos fatores de risco ao custo de capital próprio de uma empresa mediante aplicação do método baseado em análise hierárquica. In: CONGRESSO BRASILEIRO DE CUSTOS, 13, 2006, Belo Horizonte. Anais do XIII CBC. Belo Horizonte: ABCustos, 2006. CD-ROM.

CAMPOS, R.; RIBEIRO, S. E. P. Considerações para o Emprego do Método de Análise Hierárquica (AHP) em Sistemas de Custeio. In: ENCONTRO DA ASSOCIAÇÃO NACIONAL DE PÓS-GRADUAÇÃO E PESQUISA EM ADMINISTRAÇÃO, 26, 2002, Salvador. Anais do XXVI ENANPAD. Salvador: ANPAD, 2002. CD-ROM.

CERETTA, P. S.; NIEDERAUER, C. A. P. Rentabilidade e Eficiência no Setor Bancário Brasileiro. RAC, v. 5, n. 3, p. 07-26, 2001. <http://www.scielo.br/pdf/rac/v5n3/ v5n3a02.pdf $>$ 
CHARNES, A.; COOPER, W. W.; LEWIN, A. Y.; SEIFORD, L. M. Data Envelopment Analysis. 2. ed. Boston: KAP, 1994.

CHING, H. Y., MARQUES, F. e PRADO, L. Contabilidade e Finanças para não Especialistas. São Paulo: Prentice Hall, 2003.

COELLI, T.; RAO, D. S. P.; BALTESE, G. E. An Introduction to Efficiency and Productivity Analysis. Boston: KAP, 1998.

COSTA, H. G. Introdução ao Método de Análise Hierárquica: análise multicritério no auxílio à decisão. Niterói: UFF, 2002.

FREZATTI, F. The "economic paradigm" in management accounting: Return on equity and the use of various management accounting artifacts in a Brazilian context. Managerial Auditing Journal, v. 22, n. 5, p. 514-532, 2007. < http://dx.doi.org/10.1108/02686900710750784>

FREZATTI, F.; GUERREIRO, R.; AGUIAR, A. B.; GOUVÊA, M. A. Análise do Relacionamento entre a Contabilidade Gerencial e o Processo de Planejamento das Organizações Brasileiras. RAC, 2 ed. especial, p. 33-54, 2007. < http://www.scielo.br/pdf/rac/v11nspe2/ a0311ns2.pdf $>$

GALVÃO, A. M.; COGAN, S. SANTOS, G. P. Um Modelo Simplificado de Custeio a partir do ABC/AHP: Estudo e Análise em uma Empresa de Turismo. In: ENCONTRO DA ASSOCIAÇÃO NACIONAL DE PÓS-GRADUAÇÃO E PESQUISA EM ADMINISTRAÇÃO, 25., 2001, Campinas. Anais do XXV ENANPAD. Campinas: ANPAD, 2001. CD-ROM.

GASPARETTO, V. O Papel da Contabilidade no Provimento de Informações para a Avaliação do Desempenho Empresarial. Revista Contemporânea de Contabilidade, v. 01, n. 02, p. 109-122, 2004.

GOMES, L. F. A. M. Teoria da Decisão. São Paulo: Thomson Learning, 2007.

GOMES, L. F. A. M.; GOMES, C. F. S.; ALMEIDA, A. T. Tomada de Decisão Gerencial: enfoque multicritério. São Paulo: Atlas, 2002.

GOMES, L. F. A. M.; GONZALEZ-ARAYA, M. C.; CARIGNANO, C. Tomada de decisões em cenários complexos. Rio de Janeiro: Pioneira Thompson Learning, 2004.

HENDRIKSEN, E. S.; VAN BREDA, M. F. Teoria da
Contabilidade. São Paulo: Atlas, 1999.

IUDÍCIBUS, S. Análise de Balanços. 9 ed. São Paulo: Atlas, 2008.

KAPLAN, R. S.; NORTON, D. P. Estratégia em Ação. Rio de Janeiro: Campus, 1997.

KIMURA, H.; SUEN, A. S. Ferramentas de Análise Gerencial baseadas em Modelos de Decisão Multicriteriais. RAE-eletrônica, v. 2, n. 1, 2003.

KIMURA, H.; SUEN, A. S.; MORI, R. G.; ISHIKAWA, S.; HANASHIRO, D. M. M. Avaliação de Desempenho Empresarial em Novos Ambientes Competitivos através do Balanced Scorecard. In: SEMINÁRIOS EM ADMINSTRAÇÃO FEA-USP, 4, 1999, São Paulo. Anais do IV SEMEAd. São Paulo: FEA-USP, 1999. CD-ROM.

LINS, M. P. E.; MEZA, L. A. Análise Envoltória de Dados e Perspectivas de Integração no Ambiente de Apoio à Decisão. Rio de Janeiro: COPPE/UFRJ, 2000.

LYRA, R. L. C. Análise Hierárquica dos Indicadores Contábeis sob a Ótica do Desempenho Empresarial. 2008. 161 f. Tese (Doutorado em Controladoria e Contabilidade) - Departamento de Contabilidade e Atuária, Faculdade de Economia, Administração e Contabilidade, Universidade de São Paulo, São Paulo.

MACEDO, M. A. S.; FARIAS DA SILVA, F.; MELO SANTOS, R. Análise do Mercado de Seguros no Brasil: uma visão do desempenho organizacional das seguradoras no ano de 2003. Revista Contabilidade \& Finanças, Edição Especial - Atuária, 2006a.

MACEDO, M. A. S.; FARIAS DA SILVA, F.; MELO SANTOS, R. Desempenho Organizacional no Setor Bancário Brasileiro: uma aplicação da Análise Envoltória de Dados. Revista de Administração da Mackenzie, v. 7, n. 1, p. 11-44, 2006 b.

MARQUES, J. A.V. C. Análise Financeira das Empresas: liquidez, retorno e criação de valor. Rio de Janeiro: UFRJ, 2004.

MATARAZZO, D. C. Análise Financeira de Balanços: abordagem básica e gerencial. 6 ed. São Paulo: Atlas, 2003.

MATOS, D. M.; MOURA, H. J. Proposta de um Modelo para Determinação do Custo de Capital Baseado em Análise Hierárquica. RAC, v. 7, n. 4, p. 119-139, 2003. < http://www.scielo.br/pdf/rac/v7n4/v7n4a07.pdf >

NEELY, A. Measuring business performance. London: 
The Economist Books, 1998.

OMAKI, E. T. Recursos Intangíveis e Desempenho em Grandes Empresas Brasileiras: avaliações dos recursos intangíveis como estimadores de medidas de desempenho financeiras. In: ENCONTRO DA ASSOCIAÇÃO NACIONAL DE PÓS-GRADUAÇÃO E PESQUISA EM ADMINISTRAÇÃO, 29., 2005, Brasília. Anais do XXIX ENANPAD. Brasília: ANPAD, 2005. CD-ROM.

ONUSIC, L. M.; CASA NOVA, S. P. C.; ALMEIDA, F. C. Modelos de Previsão de Insolvência utilizando a Análise Envoltória de Dados: aplicação a empresas brasileiras. RAC, 2 ed. Especial, p. 77-97, 2007. < http://dx.doi. org/10.1590/S1415-65552007000600005>

PAMPLONA, E. O. Avaliação Qualitativa de Cost Drivers pelo método AHP. In: CONGRESSO BRASILEIRO DE CUSTOS, 6, 1999, São Paulo. Anais do VI CBC. São Paulo: ABCustos, 1999. CD-ROM.

PEREIRA DA SILVA, J. Análise Financeira das Empresas. 9 ed. São Paulo: Atlas, 2008.

PEREZ JR., J. H. e BEGALLI, G. A. Elaboração das Demonstrações Contábeis. 3 ed. São Paulo: Atlas, 2002.

RAFAELI, L.; MÜLLER, C. J. Estruturação de um índice consolidado de desempenho utilizando o AHP. Gestão e Produção, v. 14, n. 2, p. 363-377, 2007.

RIBEIRO, A. C.; COSTA, H. G. Emprego do Método de Análise Hierárquica (AHP) na Distribuição de Custos Indiretos: uma proposta para a pequena e média empresa. In: ENCONTRO NACIONAL DE ENGENHARIA DE PRODUÇÃO, 19, 1999, Rio de Janeiro. Anais do XIX ENEGEP. Rio de Janeiro: ABEPRO, 1999. CD-ROM.

RIBEIRO, S. E. P.; CAMPOS, R. Uma aplicação do ABC/ AHP em uma indústria de laticínios. In: CONGRESSO INTERNACIONAL DE CUSTOS, 9, 2005, Florianópolis. Anais do IX Congresso do IIC. Florianópolis: IIC, 2005. CD-ROM

SAATY, T. L. Método de Análise Hierárquica. São Paulo: McGraw-Hill/Makron, 1991.

SANCHES DA SILVA, E. R.; BORNIA, A. C.; PAMPLONA, E. O. Contribuição dos sistemas de medição de desempenho no ambiente de integração da cadeia de suprimentos. In: CONGRESSO BRASILEIRO DE CUSTOS, 13, 2006, Belo Horizonte. Anais do XIII CBC. Belo Horizonte: ABCustos, 2006. CD-ROM.

SANTOS, A.; CASA NOVA, S. P. C. Proposta de um
Modelo Estruturado de Análise de Demonstrações Contábeis. RAE-eletrônica, v. 4, n. 1, art. 8, 2005. Disponível em: <http://www.rae.com.br/eletronica>. Acesso em: 13 jan. 2008.

SHIMIZU, T. Decisão nas Organizações. São Paulo: Atlas, 2001 .

SIEGEL, S.; CASTELLAN Jr., N. J. Estatística NãoParamétrica para Ciências do Comportamento. 2 ed. Porto Alegre: Artmed, 2006.

VERGARA, S. C. Projetos e Relatórios de Pesquisa em Administração. 10. ed. São Paulo: Atlas, 2009.

VILELA, D. L.; NAGANO, M. S.; MERLO, E. M. Aplicação da Análise Envoltória de Dados em Cooperativas de Crédito Rural. RAC. 2 ed. Especial, p. 99-120, 2007. $<$ http://dx.doi.org/10.1590/S1415-65552007000600006>

YURDAKULA, M.; IÇ, Y. T. AHP approach in the credit evaluation of the manufacturing firms in Turkey. International Journal of Production Economics, v. 88, p. 269-289, 2004. <http://dx.doi.org/10.1016/S0925-5273(03)00189-0>

ZHU, J. Multi-factor Performance Measure Model with Application to Fortune 500 Companies. European Journal of Operational Research, v. 123, n. 1, p. 105-124, 2000. <http://dx.doi.org/10.1016/S0377-2217(99)00096-X> 